\title{
Prevalence and Potential Risk Factors of Helicobacter pylori Infection among Asymptomatic Individuals in Kazakhstan
}

\author{
Linda Mezmale ${ }^{1,2,3 *}$, Inese Polaka ${ }^{1}$, Dace Rudzite ${ }^{1}$, Reinis Vangravs ${ }^{1}$, Ilze \\ Kikuste $^{1,2,4}$, Sergei Parshutin', Ilva Daugule ${ }^{1,2}$, Altynbek Tazhedinov ${ }^{5}$, Tatyana \\ Belikhina $^{6}$, Nurbek Igissinov ${ }^{7,8,9}$, Jin Young Park ${ }^{10}$, Rolando Herrero ${ }^{10,11}$, Marcis \\ Leja $\mathbf{a}^{1,2,3,4}$
}

\begin{abstract}
Background: Helicobacter pylori (H. pylori) infection is associated with several risk factors such as demographic, socioeconomic status and personal habits, which vary in different populations. This is the most up-to-date data on $H$. pylori prevalence and potential risk factors for $H$. pylori infection among asymptomatic middle-aged individuals in Kazakhstan. Methods: Apparently healthy individuals aged 40 to 64, who took part in the health control in the outpatient clinic, were invited to participate in the study; answered a questionnaire, donated a blood sample. The antibodies to $H$. pylori were analysed by latex agglutination method. The baseline characteristics of study subjects with or without $H$. pylori infection were compared using the Chi-square test. Odds ratio (OR) and $95 \%$ confidence intervals (CI) for the association between $H$. pylori infection and potential risk factors were estimated using multivariable logistic regression models. Results: Altogether 166 subjects (59\% male; the median age - 51 years old) were included; 104 (62.7\%) were H. pylori positive. There were no statistically significant differences between $H$. pylori positive and H. pylori negative groups in respect to the gender, anthropometric measurements, socioeconomic factors and personal habits. The multiple variable analysis showed that age (OR, 1.99; 95\% CI, $1.03-3.86$; $\mathrm{P}=0.04)$ and increased salt intake (OR, 2.21; 95\% $\mathrm{CI}, 1.12-4.35 ; \mathrm{P}=0.02)$ were associated with $H$. pylori infection. Conclusions: More than half of the study subjects were infected with $H$. pylori in Kazakhstan. The prevalence of $H$. pylori infection was independently associated with older age and regular high salt consumption.
\end{abstract}

Keywords: Helicobacter pylori- risk factors- prevalence- Kazakhstan- gastric cancer

Asian Pac J Cancer Prev, 22 (2), 597-602

\section{Introduction}

Helicobacter pylori (H. pylori) infection is still a significant public health problem worldwide (Hooi et al., 2017), and it is the major risk factor for the development of gastric cancer (Pereira-Marques et al., 2019; Wroblewski et al., 2010). Despite a decreasing trend in incidence rates during recent decades, it is still among the most common cancers and leading causes of cancer death globally (Igissinov et al., 2018). De Martel et al., (2020) reported that 810,000 new cases of gastric cancer (mainly non-cardia gastric adenocarcinoma) were attributed to chronic H. pylori infection in 2018. In Kazakhstan, gastric cancer ranks fourth in incidence among all types of cancer (Globocan., 2018). However, the incidence of gastric cancer tends to decrease, which is associated with successful prevention of this pathology (Igissinov et al., 2018). The effective means of preventing gastric cancer in the developed world include diet, lifestyle modification, as well as successful H. pylori testing and treatment (Rawla and Barsouk, 2019).

The prevalence of $H$. pylori infection varies according to the geographic region (de Martel et al., 2018). Countries with the highest cancer incidence also have high $H$. pylori infection rates (Ferlay et al., 2015; Pereira-Marques et al., 2019). One of such countries is Kazakhstan - a multiethnic state with a diverse cultural heritage (Bray et al., 2018).

H. pylori infection is also associated with several

${ }^{I}$ Institute of Clinical and Preventive Medicine, University of Latvia, Riga, Latvia. ${ }^{2}$ Faculty of Medicine, University of Latvia, Riga, Latvia. ${ }^{3}$ Riga East University Hospital, Riga, Latvia. ${ }^{4}$ Digestive Disease Centre GASTRO, Riga, Latvia. ${ }^{5}$ Regional Diagnostic Centre, Almaty, Kazakhstan. ${ }^{6}$ Semey Regional Oncology Centre, Semey, Kazakhstan. ${ }^{7}$ Astana Medical University, Nur-Sultan, Kazakhstan. ${ }^{8}$ Central Asian Cancer Institute, Nur-Sultan, Kazakhstan. ${ }^{9}$ Eurasian Institute For Cancer Research, Bishkek, Kyrgyzstan. ${ }^{10}$ Prevention and Implementation Group, Section of Early Detection and Prevention, International Agency for Research on Cancer, Lyon, France. ${ }^{11}$ Agencia Costarricense de Investigaciones Biomédicas, San José, Costa Rica. *For Correspondence: mezmale.l@gmail.com 
risk factors - demographic, socioeconomic status, environmental and sanitation factors, as well as diet and lifestyle habits (Ozbey and Hanafiah, 2017; Travis et al., 2010). A previous Kazakhs study, revealed higher $H$. pylori positivity among persons with low educational and occupational levels, in persons who grew up in the villages and were river water drinkers, as well as among those who used outdoor facilities (Nurgalieva et al., 2002).

Previously our group published data from the same cohort suggesting that the prevalence of gastric mucosal atrophy among asymptomatic individuals in Kazakhstan was very low (Mezmale et al., 2019). However, the gastric cancer incidence was high in this area. The aim of the present study was to analyse the prevalence of $H$. pylori infection and potential risk factors among asymptomatic subjects in the population of Kazakhstan.

\section{Materials and Methods}

\section{Study design and participants}

The study was performed as a part of the regional pilot study of GISTAR (Leja et al., 2017) project in collaboration with Kazakh Institute of Oncology and Radiology, Almaty Diagnostic Centre, Semey Regional Oncology Centre.

The potentially eligible patients were identified among the outpatient clinics of the diagnostic centres in two Kazakhstan cities - Almaty and Semey. Individuals aged 40 to 64 years, who attended the clinic, were invited to participate in the study. The exclusion criteria for participants were: a personal history of gastric or colorectal cancer, gastric resection due to benign disease, self-reported or documented $H$. pylori eradication therapy in the past, antibiotic use within one month prior to the enrolment, proton pump inhibitors or bismuth-containing drug use within two weeks prior enrolment, presence of alarm symptoms for digestive or any other diseases.

A specially trained staff took blood samples, recorded the anthropometric measurements of the study participants and administered the standardized GISTAR (Leja et al., 2017) questionnaire.

\section{Sample collection and laboratory methods}

A detailed standard operating procedure for biospecimen collection has been developed according to the study protocol. A 10-ml blood sample of each participant was obtained from the antecubital vein in the morning after overnight fasting. After the blood sample was received, it was stored at $-80 \mathrm{oC}$ until the laboratory analyses were conducted. All samples were collected in regional collaborating centres in Kazakhstan and transported to Riga, Latvia for further analysis.

The antibodies to H. pylori were analysed in plasma by latex agglutination method (LZ Eiken H. pylori antibody; Eiken Chemical Co. Ltd., Tochigi, Japan). The cut-off for H. pylori positivity was $\geq 10 \mathrm{U} / \mathrm{mL}$.

Questionnaire data collection, measurement of anthropometric elements

Each study participant was interviewed by trained staff. The questionnaire captured the information regarding demographic (age, gender, ethnicity), socioeconomic characteristics (marital status, education level, household population, family income, employed status), and personal habits (regular smoking, general alcohol consumption, increased salt intake - always use extra salt with meals, intake of spicy food).

The core elements of anthropometry such as height, weight and body circumference (waist, hip) of all participants were measured. Further, a body mass index (BMI) was calculated. The BMI was classified into four categories: underweight $(<18.5)$, normal weight $(18.5-$ $24.9)$, overweight $(25.0-29.9)$, obese $(\geq 30)(\mathrm{NIH}, 2020)$.

\section{Statistical analysis}

All statistical analyses were performed using SPSS 20.0 (Statistical Package for Social Sciences, IBM Corporation, USA). The baseline characteristics of study participants with or without $H$. pylori infection were compared, using the Chi-square test. Descriptive analysis was conducted, and continuous variables were reported using mean $\pm \mathrm{SD}$; for categorical variables we used frequencies with percentages. As well as the participants were categorized by age into two groups: less or equal to 50 years and older than 51 year.

Multiple logistic regression analysis was performed to identify independent risk factors for $H$. pylori infection. Odds ratio (OR) and 95\% confidence intervals (CI) for the association between $H$. pylori infection and potential risk factors were estimated. Variables were selected for entry into the regression model if potential risk factors were significantly associated with $H$. pylori infection (P-value $<0.05)$ in the univariate analysis, and those variables which were a borderline significant $(\mathrm{P}$-value $<0.09)$. All statistical tests were two-sided and statistical significance level was set at the 5\% level.

\section{Ethical considerations}

The study protocol was reviewed and approved by the Ethics Committee of Riga East University Hospital Support Foundation - 06.10.2016., reg. No.10/16 in Riga, Latvia. Signed informed consent was obtained from all participants before enrollment in the study.

\section{Results}

Altogether 166 individuals from the general population in Kazakhstan were included from three study centres: from Kazakh Institute of Oncology and Radiology - eight (5\%) individuals; from Almaty Diagnostic Centre - 32 (19\%); from Semey Regional Oncology Centre - 126 $(76 \%)$ individuals.

\section{Demographic data and anthropometric measurements}

Out of 166 participants, 98 (59\%) were male and 68 (41\%) female; the median age of study subjects was 51 (range from 40 to 64 years). There was no statistically significant difference between gender in respect to the average age $(\mathrm{P}=0.38)$.

The majority of the study subjects - $112(67.5 \%)$ were Kazakhs, 46 (27.7\%) were Russians and 8 (4.8\%) were representatives of other ethnic groups including Chechen, 
DOI:10.31557/APJCP.2021.22.2.597

Prevalence and Risk Factors of Helicobacter pylori in Kazakhstan

Table 1. Demographic Features of Participants with and without H. pylori Infection, Univariate Analysis

\begin{tabular}{|c|c|c|c|c|c|c|}
\hline H. pylori infection & & Total positive no. & $\%$ & Total negative no. & $\%$ & P-value \\
\hline \multirow[t]{2}{*}{ Gender } & Male & 65 & 66.3 & 33 & 33.7 & 0.24 \\
\hline & Female & 39 & 57.4 & 29 & 42.6 & \\
\hline \multirow[t]{2}{*}{ Age (years) } & $\leq 50$ & 23 & 30.3 & 53 & 69.7 & 0.08 \\
\hline & $>50$ & 39 & 43.3 & 51 & 56.7 & \\
\hline \multirow[t]{3}{*}{ Ethnicity } & Kazakhs & 74 & 66.1 & 38 & 33.9 & 0.05 \\
\hline & Russians & 23 & 50 & 23 & 50 & \\
\hline & Other & 7 & 87.5 & 1 & 12.5 & \\
\hline
\end{tabular}

no, number

Table 2. Anthropometric Measurements of Participants with And without H. Pylori Infection, Univariate Analysis

\begin{tabular}{|c|c|c|c|c|c|}
\hline H. pylori infection & Total positive no. & $\%$ & Total negative no. & $\%$ & P-value \\
\hline \multicolumn{6}{|l|}{$\operatorname{BMI}\left(\mathrm{kg} / \mathrm{m}^{2}\right)$} \\
\hline$<18.5$ & 1 & 100 & 0 & 0 & \\
\hline $18.5-24.9$ & 30 & 57.7 & 22 & 42.3 & 0.64 \\
\hline $25.0-29.9$ & 44 & 67.7 & 21 & 32.3 & \\
\hline \multirow[t]{2}{*}{$>30$} & 29 & 60.4 & 19 & 39.6 & \\
\hline & Positive, mean \pm SD & & Negative, mean \pm SD & & \\
\hline Waist circumference $(\mathrm{cm})$ & $96.2 \pm 13.7$ & & $93.2 \pm 15.4$ & & 0.18 \\
\hline Hip circumference $(\mathrm{cm})$ & $106.2 \pm 10.0$ & & $106.8 \pm 12.9$ & & 0.81 \\
\hline
\end{tabular}

BMI, body mass index; SD, standard deviation; cm, centimeters, no., number

Kurdish, Tatar, Uyghur, Korean, Azerbaijanis.

H. pylori seropositivity did not differ between men and women: 65 (65.5\%) vs $39(57.5 \%)(\mathrm{P}=0.24)$. From all Kazakhs 74 (66.1\%) were H. pylori positive and this ethnic group had a higher proportion of $H$. pylori infection than other ethnic groups $(\mathrm{P}=0.05)$. Age group $>50$ years was also associated with the presence of infection. The demographic features of 166 participants with and without

Table 3. Relationships between The Prevalence of H. pylori Infection and Potential Risk Factors, Univariate Analysis

\begin{tabular}{|c|c|c|c|c|c|c|}
\hline H. pylori infection & & Total positive no. & $\%$ & Total negative no. & $\%$ & P-value \\
\hline \multirow[t]{2}{*}{ Living in a household } & Alone & 3 & 42.9 & 4 & 57.1 & 0.26 \\
\hline & With family members & 100 & 63.7 & 57 & 36.3 & \\
\hline \multirow[t]{2}{*}{ Family status } & Married & 85 & 63 & 50 & 37 & 0.96 \\
\hline & Single & 19 & 63.3 & 11 & 36.7 & \\
\hline \multirow[t]{2}{*}{ Number of children } & $\leq 2$ & 76 & 61.8 & 47 & 38.2 & 0.69 \\
\hline & $>2$ & 28 & 65.1 & 15 & 34.9 & \\
\hline \multirow[t]{2}{*}{ Siblings } & $\leq 2$ & 45 & 63.4 & 26 & 36.6 & 0.86 \\
\hline & $>2$ & 59 & 62.1 & 36 & 37.9 & \\
\hline \multirow[t]{2}{*}{ Education level } & Basic & 70 & 63.1 & 41 & 36.9 & 0.87 \\
\hline & Higher & 34 & 61.8 & 21 & 38.2 & \\
\hline \multirow[t]{2}{*}{ Employed status } & Unemployed & 79 & 66.4 & 40 & 33.6 & 0.11 \\
\hline & Employed & 25 & 53.2 & 22 & 46.8 & \\
\hline \multirow[t]{2}{*}{ Family income per month } & $\leq 100 €$ & 65 & 60.2 & 43 & 39.8 & 0.33 \\
\hline & $>100 €$ & 38 & 67.9 & 18 & 32.1 & \\
\hline \multirow[t]{2}{*}{ Alcohol drinking } & Yes & 50 & 61 & 32 & 39 & 0.65 \\
\hline & No & 54 & 64.3 & 30 & 35.7 & \\
\hline \multirow[t]{2}{*}{ Cigarette smoking } & Yes & 67 & 66.3 & 34 & 33.7 & 0.22 \\
\hline & No & 37 & 56.9 & 28 & 43.1 & \\
\hline \multirow[t]{2}{*}{ Intake of spicy food } & Yes & 33 & 66 & 17 & 34 & 0.55 \\
\hline & No & 71 & 61.2 & 45 & 38.8 & \\
\hline \multirow[t]{2}{*}{ Increased salt intake } & Yes & 54 & 56.3 & 42 & 43.7 & 0.04 \\
\hline & No & 50 & 71.4 & 20 & 28.6 & \\
\hline
\end{tabular}


Table 4. Multiple Logistic Regression Model for Predicting the Risk for H. pylori Infection

\begin{tabular}{llccc}
\hline H. pylori infection & & Adjusted OR & $95 \%$ CI & P-value \\
\hline Increased salt intake & Yes & 2.21 & $1.12-4.35$ & 0.02 \\
Age group & No & 1 & - & - \\
& $>50$ & 1.99 & $1.03-3.86$ & 0.04 \\
Nationality & $\leq 50$ & 1 & - & - \\
& Other & 0.28 & $0.03-2.44$ & 0.24 \\
& Russian & 1.64 & $0.79-3.40$ & 0.18 \\
& Kazakhs & 1 & - & - \\
\hline
\end{tabular}

$\overline{\mathrm{OR}}$, odds ratio, $\mathrm{CI}$, confidence interval.

H. pylori infection are summarized in Table 1.

Anthropometric measurements of the two study groups are summarized in Table 2. There were no statistically significant differences between $H$. pylori positive and negative groups based on anthropometric measurements $(\mathrm{P}>0.05)$.

Socioeconomic characteristics and personal habits

The socioeconomic features and personal habits of study subjects in relation to the presence of $H$. pylori infection are summarised in Table 3. No significant difference was observed between socioeconomic factors and $H$. pylori prevalence $(\mathrm{P}>0.05)$. However, participants who reported increased salt consumption were more likely to be $H$. pylori positive $(\mathrm{P}=0.04)$.

\section{Prediction of $H$. pylori infection risk}

The final multiple logistic regression model included the following variables - increased salt intake, age groups and nationality (Table 4). Only increased salt consumption and age group older than 50 years were significantly associated with infection.

\section{Discussion}

To our knowledge, this is the most up-to-date information on prevalence and potential risk factors for H. pylori infection among asymptomatic individuals in Kazakhstan.

Since the incidence of gastric cancer in Kazakhstan is high and ranks at the third-place from all cancers (Globocan, 2018), a high prevalence of $H$. pylori infection was expected. The idea was supported by our results showing that the overall prevalence of $H$. pylori infection in the study population was $62.7 \%$. However, previous studies in Kazakhstan have demonstrated even higher infection rate: the overall prevalence of $H$. pylori seropositivity in 2010 among asymptomatic adults was $86 \%$ and 64\% among children (Benberin et al., 2013), while in symptomatic population $H$. pylori infection was found to be $62 \%$ in 2002 (Nurgalieva et al., 2002). Nevertheless, the prevalence of infection in Kazakhstan in our study was higher than in the neighbouring countries: the latest data show that the prevalence of $H$. pylori infection in Kyrgyzstan was 51,9\% (Dzhumabaev et al., 2015), in China - 52,2\% (W. Wang et al., 2019), and in Russia - 53\% (Plavnik RG, 2019). Higher infection rate has been reported only in Uzbekistan, where the prevalence of $H$. pylori infection was $74.9 \%$ (Abdiev et al., 2010). It should be noted, that both countries Kazahstan and Uzbekistan showed a high incidence of gastric cancer in 2018: in Uzbekistan incidence rate of gastric cancer was 10,4 per 100000 population, meanwhile, in Kazakhstan - 15,7 per 100000 inhabitants (Globocan, 2018).

Increased age is generally accepted as a risk factor for $H$. pylori infection and many studies report increasing prevalence of $H$. pylori infection with increasing age (S. Chen et al., 2013; Hirayama et al., 2014; Syam et al., 2015; W. Wang et al., 2019). Similarly, our team found that $H$. pylori infection was more likely in an age group older than 50, and the age was a significant predictor for $H$. pylori infection. In the previous study conducted in 2002 by Kazakhstan scientists have reported that the $H$. pylori prevalence increases from 40 years onwards and the highest prevalence was found in the age group of 50-59 (Nurgalieva et al., 2002).

Although univariate analysis showed a significantly higher prevalence of $H$. pylori among Kazakhs compared to Russians, in the multivariate analysis ethnicity was not found to be a significant predictor for $H$. pylori infection. Similarly, Nurgalieva and team did not find any association between $H$. pylori infection Kazakhs and Russians (Nurgalieva et al., 2002).

Possible factors that could interact with ethnicity in the statistical analysis could be dietary habits. In our study, increased salt intake was a significant risk factor for $H$. pylori infection in the multivariate analysis. We have to note that $57,8 \%$ of study participants reported use of extra salt with meals. According to the World Health Organization (WHO) data, salt intake in Kazakhstan stands at about 17 grams per day, which is more than three times above the WHO recommended daily intake (WHO, 2019). It could be related to traditional cooking - people use salt not only for meat and sausages, but also for traditional yoghurt products such as kurt and airan (WHO, 2019). H. pylori infection together with increased salt consumption may play an important role in gastric cancer development. For example, salty food could cause temporary damage of the gastric mucosa leading to changes in the viscosity of the gastric mucosal barrier in that way facilitating $H$. pylori colonization and increased risk of developing gastric cancer (Nozaki et al., 2003). Similarly, a study from China demonstrated that patients with increased salt consumption and $H$. pylori have a higher risk of developing gastric cancer (Zhong et al., 2012). A large review of epidemiological studies 
performed by Wang et al. confirmed a positive association between increased intake of salt and the risk of gastric cancer (Wang et al., 2009). Possibly, high salt diet together with $H$. pylori might explain the high incidence of gastric cancer in Kazakhstan.

Although several studies show an association of $H$. pylori infection with socioeconomic factors, smoking and alcohol consumption (Chen et al., 2014; Wang et al., 2019), none of the studied factors (marital status, education level, employment, smoking and alcohol consumption) in our population sample was related to H. pylori infection. The same results were reported by Ogihara et al. (Ogihara et al., 2000) and Shinchi et al. (Shinchi et al., 1997) suggesting that these factors are not independent predictors for $H$. pylori infection. Similarly, no significant association was found between $H$. pylori infection and anthropometric measurements (height, weight and body circumference) although several previous studies show that patients with overweight or obesity are more likely to have $H$. pylori infection (Al-Zubaidi et al., 2018; Lender et al., 2014; Xu et al., 2014).

It should be noted that the study has limitations. The main limitation was the small study population size, thus reducing the statistical power of the study. Despite the proportional distribution between ethnic groups, it would be worthwhile to increase the study population size, to make the results more reliable.

In conclusion, the results of the study demonstrate that the overall prevalence of $H$. pylori infection among asymptomatic middle-aged subjects in Kazakhstan was $62.7 \%$ and $H$. pylori infection is independently associated with older age and increased salt consumption. However, factors such as anthropometric measurements, socioeconomic factors were not related to $H$. pylori infection in the studied population. However, it should be noted that we were not able to fully assess this population due to the aforementioned limitations.

Knowledge of the risk factors of $H$. pylori infection is important because it could prevent the spread of the bacterium in a high-risk population like Kazakhstan, which has a high burden of gastric cancer.

\section{Acknowledgments}

\section{Funding Statement}

The work was supported by FLPP (Fundamental and Applied Research Projects) Programm in Latvia, project nr. lzp-2018/1-0135 "Research on implementation of a set of measures for prevention of gastric cancer mortality by eradication $H$. pylori and timely recognition of precancerous lesions" and Ministry of Education and Science of the Republic of Kazakhstan, project nr. AP05133849 "Decreasing the burden of gastric cancer in Kazakhstan: evaluation of the existing situation and search for improvement possibilities".

\section{Authors' contributions}

All authors contributed to the study conception and design. Data collection and preparation for analyses by Altynbek Tazhedinov, Ilze Kikuste, Tatyana Belikhina, Nurbek Igissinov; data analysis by Dace Rudzite, Reinis
Vangravs, Linda Mezmale, Inese Polaka; technical data storage and technical support - Sergei Parshutin; data interpretation: all authors; writing of the first draft: Linda Mezmale, Marcis Leja; revised and approved by all authors.

Statement conflict of interest

The authors declare no conflicts of interest.

\section{References}

Abdiev S, Ahn KS, Khadjibaev A, et al (2010). Helicobacter pylori infection and cytokine gene polymorphisms in Uzbeks. Nagoya J Med Sci, 72, 167-72.

Al-Zubaidi AM, Alzobydi AH, Alsareii SA, et al (2018). Body massindex and Helicobacter pylori among obese and nonobese patients in Najran, Saudi Arabia: A Case-Control Study. Int J Environ Res Public Health, 15, 2586.

Benberin V, Bektayeva R, Karabayeva R, et al (2013). Prevalence of $H$. pylori infection and atrophic gastritis among symptomatic and dyspeptic adults in Kazakhstan. A hospital-based screening study using a panel of serum biomarkers. Anticancer Res, 33, 4595-602.

Bray F, Ferlay J, Soerjomataram I, et al (2018). Global cancer statistics 2018: GLOBOCAN estimates of incidence and mortality worldwide for 36 cancers in 185 countries. $C A$ Cancer J Clin, 68, 394-424.

Chen HL, Chen MJ, Shih SC, et al (2014). Socioeconomic status, personal habits, and prevalence of Helicobacter pylori infection in the inhabitants of Lanyu. J Formos Med Assoc, 113, 278-83.

Chen S, Ying L, Kong M, Zhang Y, Li Y (2013). The prevalence of Helicobacter pylori infection decreases with older age in atrophic gastritis. Gastroenterol Res Pract, 2013, 494783

de Martel C, Georges D, Bray F, Ferlay J, Clifford G M (2020). Global burden of cancer attributable to infections in 2018: a worldwide incidence analysis. Lancet Glob Health, 8 , e180-90.

Dzhumabaev MN, Dzhumanova RG, Sabirov IS (2015). The interdependence between smoking, alcohol, tooth pathology and prevalence of helicobacter pylori among ethnic groups in kyrgyzstan. Eksp Klin Gastroenterol, 6, 16-20.

Ferlay J, Soerjomataram I, Dikshit R, et al (2015). Cancer incidence and mortality worldwide: sources, methods and major patterns in GLOBOCAN 2012. Int J Cancer, 136, E359-86.

Globocan (2018). https://gco.iarc.fr/today/data/factsheets/ populations/398-kazakhstan-fact-sheets.pdf. Accessed May 5, 2020.

Hirayama Y, Kawai T, Otaki J, Kawakami K, Harada Y (2014). Prevalence of Helicobacter pylori infection with healthy subjects in Japan. J Gastroenterol Hepatol, 29, 16-9.

Hooi J KY, Lai WY, Ng WK, et al (2017). Global Prevalence of Helicobacter pylori Infection: Systematic Review and Meta-Analysis. Gastroenterology, 153, 420-9.

Igissinov N, Kozhakhmetov S, Malayev NB, et al (2018). Evaluation gastric cancer incidence in Kazakhstan. 195, 30-6.

Leja M, Park JY, Murillo R, et al (2017). Multicentric randomised study of Helicobacter pylori eradication and pepsinogen testing for prevention of gastric cancer mortality: the GISTAR study. BMJ Open, 7, e016999

Lender N, Talley NJ, Enck P, et al (2014). Review article: Associations between Helicobacter pylori and obesity-an ecological study. Aliment Pharmacol Ther, 40, 24-31.

Mezmale L, Isajevs S, Bogdanova I, et al (2019). Prevalence 
of atrophic gastritis in Kazakhstan and the accuracy of pepsinogen tests to detect gastric mucosal atrophy. Asian Pac J Cancer Prev, 20, 3825-9.

National Heart, Lung and Blood Institute. https://www.nhlbi.nih. gov/health/educational/lose_wt/BMI/bmicalc.htm. Accessed May 5, 2020.

Nozaki K, Tsukamoto T, Tatematsu M (2003). Effect of high salt diet and Helicobacter pylori infection on gastric carcinogenesis. Nihon Rinsho, 61, 36-40.

Nurgalieva ZZ, Malaty HM, Graham DY, et al (2002). Helicobacter pylori infection in Kazakhstan: effect of water source and household hygiene. Am J Trop Med Hyg, 67, 201-6.

Ogihara A, Kikuchi S, Hasegawa A, et al (2000). Relationship between Helicobacter pylori infection and smoking and drinking habits. J Gastroenterol Hepatol, 15, 271-6.

Ozbey G, Hanafiah A (2017). Epidemiology, diagnosis, and risk factors of Helicobacter pylori infection in children. Euroasian J Hepato Gastroenterol, 7, 34-9.

Pereira-Marques J, Ferreira RM, Pinto-Ribeiro I, Figueiredo C (2019). Helicobacter pylori Infection, the Gastric Microbiome and Gastric Cancer. Eds Kamiya S, Backert S. Helicobacter pylori in Human Diseases: Advances in Microbiology, Infectious Dis Public Health, 11, 195-210

Plavnik RG, Mareyeva DV, Bordin DS (2019). Helicobacter pylori Epidemiology: Clinical and Laboratory Parallels. Effective pharmacotherapy. Gastroenterology, 15, 16-20.

Rawla P, Barsouk A (2019). Epidemiology of gastric cancer: global trends, risk factors and prevention. Przeglad Gastroenterol, 14, 26-38.

Shinchi K, Ishii H, Imanishi K, Kono S (1997). Relationship of cigarette smoking, alcohol use, and dietary habits with Helicobacter pylori infection in Japanese men. Scand $J$ Gastroenterol, 32, 651-5.

Syam AF, Miftahussurur M, Makmun D, et al (2015). Risk factors and prevalence of Helicobacter pylori in five largest Islands of Indonesia: A Preliminary Study. PLoS One, 10, $\mathrm{e} 0140186$.

Travis PB, Goodman KJ, O'Rourke KM, et al (2010). The association of drinking water quality and sewage disposal with Helicobacter pylori incidence in infants: the potential role of water-borne transmission. J Water Health, 8, 192-203.

Wang W, Jiang W, Zhu S, et al (2019). Assessment of prevalence and risk factors of helicobacter pylori infection in an oilfield Community in Hebei, China. BMC Gastroenterol, 19, 186.

Wang X-Q, Terry P-D, Yan H (2009). Review of salt consumption and stomach cancer risk: epidemiological and biological evidence. World J Gastroenterol, 15, 2204-13.

World Health Organization (2019). https://www.euro.who.int/ en/countries/kazakhstan/news/news/2019/03/who-studiesreveal-kazakhstan-has-among-the-highest-levels-of-saltintake-globally. Accessed May 5, 2020.

Wroblewski LE, Peek RM Jr, Wilson KT (2010). Helicobacter pylori and gastric cancer: factors that modulate disease risk. Clin Microbiol Rev, 23, 713-39.

Xu C, Yan M, Sun Y, et al (2014). Prevalence of Helicobacter pylori infection and its relation with body mass index in a Chinese population. Helicobacter, 19, 437-42.

Zhong C, Li KN, Bi JW, Wang BC (2012). Sodium intake, salt taste and gastric cancer risk according to Helicobacter pylori infection, smoking, histological type and tumor site in China. Asian Pac J Cancer Prev, 13, 2481-4.

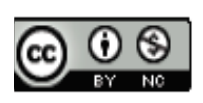

This work is licensed under a Creative Commons AttributionNon Commercial 4.0 International License. 